\title{
Human otoacariasis
}

\author{
K. S. Gangadhara Somayaji · A. Rajeshwari
}

\begin{abstract}
Accidental entry of foreign bodies into the ear canal is very common. Animate foreign bodies constitute upto $14 \%$ of cases, majority being the cockroaches. Not many cases of ticks entering into human ears are found in the scientific literature. Even the available reports are from South Africa, Nepal, Malaysia, Chile and Srilanka. This Indian study discusses the occurence, clinical features, the methods adopted in the removal and the complications of tick infestation of human ear. A total of 144 cases of ticks entering the human ears were studied over a period of two years from Jan 2004 to Dec 2005.This report represents one of the largest recorded series of human otoacariasis available in the Indian literature.
\end{abstract}

Keywords Otoacariasis · Ticks $\cdot$ Human

K. S. G. Somayaji ${ }^{1}(\bowtie) \cdot$ A. R. Sharada ${ }^{2}$

${ }^{1}$ Associate Professor in ENT,

${ }^{2}$ Ex Associate Professor,

(Presently working as Reader in ENT at Yenepoya Medical College,

Mangalore)

K.V.G Medical College Hospital, Sullia

Karnataka - 574327

Sharada Vasudeva Sadana

No: 3-3/88, $3^{\text {rd }}$ main road,

Prashanth Nagara Layout,

Behind Derebail church, Mangalore

Karnataka - 575006

email: ksgsomayaji@yahoo.co.in; ksgsomayaji@gmail.com

Ph: +91 / 824 / 2240258 M: +91 / 9448150448

\section{Introduction}

Accidental entry of arthropods [insects] particularly, cockroaches is a common cause of live foreign bodies in the ear [1]. Otoacariasis meaning the presence of ticks and mites in the ear canal of animals other than man is a well recognized problem. Human Otoacariasis is observed in and around the western ghat area. Removing these live ticks from the ear canal can be challenging even for ENT surgeons as ticks are highly resistant to most of the insecticidal reagents [2]. The aims of this publication are to report the occurrence, pattern of distribution, clinical presentation, problems encountered in the removal of these ticks and the reagents used.

\section{Materials and methods}

This study was conducted over a period of two years from Jan 2004 to Dec 2005 at K.V.G Medical College, Sullia of Mangalore district in Karnataka state which is situated at the base of Western Ghats. All the patients who came with animate foreign bodies in the ear canal were included in the study. Only the patients who had tick in the ear were considered for further study. The clinical manifestations in these patients and the management are discussed in this paper.

\section{Ohservations}

A total number of 144 cases presented with animate intraaural foreign bodies during the period of study from Jan 2004 to Dec 2005. Out of this, 126 patients had tick in their ears. (Table 1). The youngest patient was one year old and the oldest was seventy years. (Table 2) Maximum numbers of cases were seen between $31-40$ years. [25 cases]. Females were affected more compared to males (Table 2).

All the patients presented with pain in the ear. 16 patients [12.7\%] also had minimal bleeding from the ear. 
Table 1 The occurence of animate foreign bodies in the ear. From Jan 2004 to Dec 2005

\begin{tabular}{lc}
\hline Animate F.B & No. \& \% of cases \\
\hline Tick & $126[87.5 \%]$ \\
Cockroach & $06[4.17 \%]$ \\
Ant & $04[2.78 \%]$ \\
Other insects & $08[5.55 \%]$ \\
\hline Total & 144 \\
\hline
\end{tabular}

Table 2 Age and Sex distribution of patients with tick infestation in the ear

\begin{tabular}{llll}
\hline Age group & No. of patients & Males & Females \\
\hline $0-10$ & 28 & 16 & 12 \\
$11-20$ & 16 & 08 & 08 \\
$21-30$ & 22 & 09 & 13 \\
$31-40$ & 25 & 10 & 15 \\
$41-50$ & 14 & 08 & 06 \\
$51-60$ & 13 & 05 & 08 \\
$61-70$ & 08 & 03 & 05 \\
\hline Total & 126 & 59 & 67 \\
\hline
\end{tabular}

Table 3 Time interval between entry of tick \& presentation

\begin{tabular}{lc}
\hline Time taken to present & No. \& $\%$ of cases \\
\hline $1^{\text {st }}$ day & $08[6.35 \%]$ \\
$2^{\text {nd }}$ day & $24[19.05 \%]$ \\
$3^{\text {rd }}$ day & $33[26.18 \%]$ \\
$4^{\text {th }}$ day & $27[21.43 \%]$ \\
$5^{\text {th }}$ day & $16[12.70 \%]$ \\
$6^{\text {th }}$ day & $10[7.94 \%]$ \\
7 days $\&$ beyond & $08[6.35 \%]$ \\
\hline Total & $126[100 \%]$ \\
\hline
\end{tabular}

Average time of presentation of the patients after the onset of symptoms is 3-4 days (Table 3 ).

The tick was found attached to the ear canal in 109 cases. [86.5\%].In the remaining 17 cases $(13.5 \%)$ it was found attached to the tympanic membrane. On otoscopic examination of these cases, the blood filled bloated body of the tick was seen resembling a hemorrhagic vesicle. In all the patients only one tick was seen and the tympanic membrane was intact in all the cases.

We studied three types of reagents in the removal of these ticks. 4\% Xylocaine solution and 4\% Xylocaine with equal quantity of turpentine was used in 30 cases each. 10\% Xylocaine spray was used in 64 cases. For 2 cases general anesthesia was used as one was very uncooperative and the other was a mentally retarded adolescent. What ever be the solution used, the patients were made to lie with the affected ear up for about 15-20 minutes. Using aural foreign body removal forceps the tick was removed from the ear canal. The debris [fecal matter of the tick] in the canal was removed by syringing. $90 \%$ of the patients (27 cases) in whom the turpentine was used, had severe pain and local reaction in the canal. Though, there were no local reactions with both $4 \%$ and $10 \%$ Xylocaine, the ease of removal and the patient comfort was significantly better with $10 \%$ Xylocaine. All the patients experienced pain during and for a variable period after the removal. The pain was unbearable when the xylocaine was not used or used for a lesser time. Prophylactic antibiotic ear drops were given to patients who had minimal inflammatory changes in the canal. One patient developed ASOM after the tick removal and was successfully treated with oral antibiotics. None of the patients had tympanic membrane perforation or facial palsy.

\section{Discussion}

Otoacariasis in animals other than man is well recognized problem. Ticks parasitize a wide range of domestic and wild hosts although this apparent preference may be due to availability rather than choice [7]. The unfed larvae of the ticks are predominantly found in forest habitats. Newly emerged larvae in such habitats will attack to a host after dropping onto it from overhanging leaves, after clinging to it as it passes by, or after climbing on it as is resting [1]. Most of the ticks attach to the head, often to the ear canal, ear drum, ear lobe and eyelid [1].

Man occasionally gets infested particularly during dry season from October to February Children and women are affected more probably due to co exposure of these two groups during sleeping together at night or due to young ones accompanying their female guardians during daily activities within or outside the domestic premises [6].In children, it could be due to their close contact with pet animals.

The most common complaint of the intraaural tick infestation is ear pain [90\%]. A review of literature also showed some of the patients coming with bleeding $(10 \%)$, giddiness $(5 \%)$, tinnitus $(5 \%)$ or facial paresis $(5 \%)$ [1]. The average time duration between the onset of symptoms and the time of presentation in our study is 3-4 days though in literature review, we found presenting after several weeks [1]. Immediate presentation as seen in other aural foreign bodies like cockroach and other insects was not observed in tick infestation. The ticks attach to the canal skin or ear drum by means of mouth parts. The enzyme contained in their saliva causes local inflammation and pain [1]. The rostrum is burrowed into the skin to enable it to suck blood. As the tick feeds, it gradually becomes engorged. At the same time, saliva is secreted which contains toxins. The neurotoxin interferes with the synthesis or liberation of 
acetylcholine at the motor end points and there by it can cause facial paralysis or respiratory paralysis In most cases of tick paralysis, the symptoms are believed to be caused by female ticks. A single female can completely paralyse and kill an adult human. Ticks can also transmit a variety of diseases like tick typhus, Rocky Mountain spotted fever, Kyasanur forest disease [K.F.D] etc.Our place is also an endemic area for the KFD.

Intraaural ticks can cause otitis externa, tympanic membrane perforation, otitis media and facial palsy. Most of the aural complications are caused by overenthusiastic attempts at the removal by inexperienced clinicians. We have not seen any case of tick infestation in discharging ears. $90 \%$ of the patients had varying degrees of external ear inflammation.

The ticks are highly resistant to most of the reagents used to kill the intraaural foreign body [2]. Lignocaine in concentrations of $4 \%$ or $10 \%$ is ideal as it paralyses the ticks and also has some anesthetic effect over the tick bite area of the canal skin. Oil of turpentine causes severe local irritation. We have not used other preparations like spirit ear drops as done by some authors [3].

Insect aural foreign bodies account for around 14\% of all foreign bodies in the ear, out of which cockroaches account for $78 \%$ of cases. High incidence of ticks in our study is probably due to more number of referral cases to the Hospital and also to the near by dense forest area.

\section{Conclusion}

Reports of tick infestation of human ears are less common in the scientific literature. Ticks can be commonest foreign body in the ear as in our case especially in tropical places. In this context, one must suspect intraaural ticks in patients coming with otalgia from dense forest areas. Doctors should be trained in proper techniques of removal in order to avoid trauma to the canal and patient discomfort. From the public health aspect, those living in the forest areas should be made aware that keeping farm animals close to them is a health hazard.

\section{References}

1. Indudharan R, Ahamed TMHo, Salim R et al (1999) Human Otoacariasis: Annals of Tropical Medicine and parasitology 93(2):163-167

2. Patrick JA, Anis BS, Angela BS (2001) Insecticidal activity of common reagents for insect foreign bodies of the ear. Laryngoscope 111(1):15-20

3. Fegan D, Glennon J (1986) Intra aural ticks in Nepal. Lancet 348:1313

4. Indudharan R, Dharap AS, HoTM (1986) Intra aural ticks causing facial palsy. Lancet 348:613

5. Dilrukshi PRMP, Yasawardene ADKSN, Amarasinghe PH et al (2004) Human Otoacariasis: A retrospective study from an area of Srilanka. Transactions of the royal society of Tropical Medicine \& Hygiene 98:489-495

6. Entomology of ticks: Suborder Ixoidida: From Text book of Medical Acarology and Entomology, Pp 1654-1659 